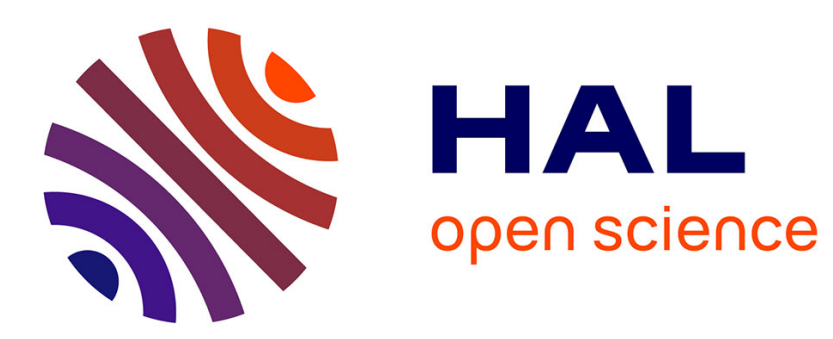

\title{
Faire vivre et mourir les institutions. Les congrégations soumises au verdict du Conseil d'État (1900-1904)
}

Antoine Perrier

\section{To cite this version:}

Antoine Perrier. Faire vivre et mourir les institutions. Les congrégations soumises au verdict du Conseil d'État (1900-1904). Revue historique, 2019, n689 (1), pp.57. 10.3917/rhis.191.0057 . hal03111136v1

\section{HAL Id: hal-03111136 \\ https://hal.science/hal-03111136v1}

Submitted on 15 Jan 2021 (v1), last revised 21 Jan 2021 (v2)

HAL is a multi-disciplinary open access archive for the deposit and dissemination of scientific research documents, whether they are published or not. The documents may come from teaching and research institutions in France or abroad, or from public or private research centers.
L'archive ouverte pluridisciplinaire HAL, est destinée au dépôt et à la diffusion de documents scientifiques de niveau recherche, publiés ou non, émanant des établissements d'enseignement et de recherche français ou étrangers, des laboratoires publics ou privés. 
Antoine Perrier, « Une chorégraphie pour rien. La communication politique lyautéenne et la distribution des pensions militaires au Maroc après la Première Guerre mondiale ", Outre-Mers, Revue d'histoire, t. 106, n ${ }^{\circ}$ 400-401, 2018, p. 237-257.

\title{
Une chorégraphie pour rien. La communication politique lyautéenne et la distribution des pensions militaires après la Première guerre mondiale
}

\section{Résumé}

Après la Première Guerre mondiale, le Protectorat marocain est appelé à distribuer des pensions militaires aux Marocains anciens combattants ou à leurs ayants droit s'ils sont décédés. L'application de cette politique rencontre des difficultés pratiques, liées à l'incompatibilité apparente entre la «coutume musulmane » et le droit français. La Grande Guerre présente alors un paradoxe particulièrement prononcé. Ce moment de grande communication politique sur le thème de l'union de la France et du Makhzen révèle, dans son immédiate suite, les difficultés de médiation de l'État colonial avec la population marocaine. Cet article propose donc de nuancer la portée du discours lyautéen en scrutant l'application d'une politique publique destinée à récompenser les Marocains pour leur engagement au service de la France. La communication lyautéenne devient un moyen de dissimulation de l'impossible médiation des autorités coloniales avec la population marocaine.

\begin{abstract}
After World War I, the French Protectorate on Morocco had to provide military pensions to Moroccan soldiers, or to their families. Implementing these pensions became hugely challenging, allegedly due to « Muslim customs ». These customs are actually a French avatar which aims to hide colonial difficulties to deal with social politics among Moroccan people. French Residence denied to Sherifian Monarchy the ability or the right to implement pensions, but could not succeed in substituting itself to traditional institutions. This case-study shows consequently to what extent the Lyautey discourse hided political inabilities of the colonial State.
\end{abstract}

\section{Notice biographique}

Antoine Perrier est agrégé d'histoire et ATER à la faculté des lettres de Sorbonne Université. Sa thèse, préparée au Centre d'histoire de Sciences Po, porte sur l'administration des monarchies marocaine et tunisienne sous le Protectorat à partir, entre autres, de leurs sources en arabe.

\author{
Mots-clés \\ Protectorat / Protectorate \\ Maroc / Morocco \\ Lyautey \\ Makhzen \\ Pension militaire / Military pension
}


«Ce serait une si périlleuse illusion d'imaginer que nous tiendrons indéfiniment (l'élite indigène) en mains avec notre mince et fragile pellicule d'occupation ${ }^{1}$. Dans cette phrase qui clôt l'examen de conscience théâtral du général Lyautey, connu comme la circulaire du coup de barre de 1920, le Résident général fait à nouveau démonstration de ses talents prophétiques. S'inquiétant, au long de cette note, d'un vent nouveau qui viendrait la menacer, Lyautey met en relief la superficialité de la présence française. La politique des pensions qu'elle pratiqua en offre un exemple : son application contrariée donne raison au «maréchal de l'Islam $»^{2}$ tout en montrant les faiblesses de son système érigé en doctrine.

La Première Guerre mondiale a la particularité d'être le moment le plus virtuose de la mise en scène par Lyautey de la collaboration avec les élites marocaines tout en donnant lieu aux discriminations et difficultés les plus évidentes du système colonial. C'est ce contraste qu'il s'agit s'interroger en scrutant d'abord les formes de communication politique de Lyautey, rapportée ici à un moment de ballet particulièrement gracieux ${ }^{3}$. Ce spectacle déjà étudié par Daniel Rivet en général ${ }^{4}$, réclame ainsi la participation des élites marocaines généralement évoquée de façon plus allusive par l'historiographie ${ }^{5}$. La Première Guerre mondiale est ainsi envisagée à travers une instrumentalisation politique qui renforce, du point de vue colonial, le Protectorat comme régime, malgré le sort parfois misérable des soldats marocains au front ${ }^{6}$. Il existe néanmoins des limites dans l'application de la politique lyautéenne et l'analyse des conséquences au long cours des politiques conflictuelles des pensions versées aux anciens combattants permet de les saisir avec clarté.

\footnotetext{
${ }^{1}$ Centre des Archives diplomatiques de Nantes (ci-après CADN,) 1/TU/1V/60, Note du général Lyautey dit «circulaire du coup de barre », 18 novembre 1920. Cette circulaire constitue un des premiers « examens de conscience » du Résident général en proposant une lecture rétrospective et critique de la politique du Protectorat. Elle souligne notamment le rôle de figurant du Makhzen et la mainmise de Paris sur la politique marocaine.

${ }^{2}$ Daniel Rivet, «Quelques propos sur la politique musulmane de Lyautey au Maroc (1912-1925) », dans PierreJean Luizard (dir.), Le choc colonial et l'islam, Paris, La Découverte, 2006, p. 259.

${ }^{3}$ Nous reprenons ici l'expression de chorégraphie employée par Abdallah Hammoudi pour décrire la dimension spectaculaire, très bien rythmée, parfaitement calculée, de la politique de communication lyautéenne. Abdallah Hammoudi, "Eléments d'anthropologie des monarchies à partir de l'exemple marocain », Rémy Leveau, Abdallah Hammoudi, (dir.), Monarchies arabes. Transitions et dérives dynastiques, La Documentation Française, Paris, 2002, p. 57.

${ }^{4}$ Daniel Rivet, Lyautey et l'institution du protectorat français au Maroc (1912- 1925), Paris, L'Harmattan, 1988.

${ }^{5}$ Mohammed Hatimi parle de façon très juste d'une tentative de «conquête des cœurs » de Lyautey, qu'il envisage selon les archives coloniales. Mohammed Hatimi, «À la conquête de "l'âme marocaine": l'économie de la force et la force de l'économie en attendant la fin des hostilités en Europe », Hespéris-Tamuda, LIII (1), 2018, p. 27-45.

${ }^{6}$ Mohamed Bekraoui, «Le Maroc et la Première Guerre mondiale : 1914-1920 », thèse d'histoire sous la direction de Jean-Louis Miège, Université d'Aix-Marseille, 1987.
} 
La distribution des pensions d'anciens combattants ou de leurs ayants droits présente la particularité d'introduire de l'argent métropolitain dans les finances autonomes du Protectorat marocain : le Trésor français rémunère les pensions des citoyens français tout comme des sujets marocains mobilisés sous les drapeaux ${ }^{7}$. Surtout, elle se manifeste comme une urgence politique, un devoir moral, une nécessité pour les anciens militaires marocains et leurs familles. Ce faisant, en obligeant les autorités coloniales à distribuer des pensions selon des règles comptables métropolitaines dans les provinces les plus reculés de l'empire chérifien, cette politique publique illustre le paradoxe du Protectorat. Durant les années 1920 et 1930, les hauts-fonctionnaires et les militaires français vont buter sur une catégorie qu'ils ont, selon l'esprit de Lyautey, forgée eux-mêmes : la « coutume musulmane », forme inviolable et sacrée des traditions à maintenir au Maroc au nom du Protectorat. Celle-ci, fondée essentiellement sur l'image d'un mode de vie reposant sur une référence religieuse, rend l'application du droit français impossible au Maroc. Elle suppose que le Marocain vit selon le Coran, a une famille polygame nécessairement innombrable, qu'il ne se marie pas et ne meurt pas comme un Français pourvu d'un état civil. Au motif de la respecter, les services coloniaux sont incapables de délivrer les pensions en bonne équité avec les anciens combattants français.

En partant de cet exemple de politique publique, il ne s'agit pas de se borner à un retour iconoclaste sur le jeu d'illusion lyautéenne mais de tenter de comprendre ce que dissimule cet obstacle construit de la coutume musulmane. Notre hypothèse est que ce motif est une façon de masquer, de façon plus ou moins réfléchie, la difficulté de mener à bien la tâche de médiation éprouvée par les autorités coloniales envers une population marocaine qui lui reste inaccessible. Cet exemple permet de discuter, à tout le moins, une historiographie prenant Lyautey au pied de la lettre ${ }^{8}$. Au contraire de bien de ses discours, le Protectorat refuse de collaborer avec les institutions de la monarchie chérifienne qui sont susceptibles de connaître les sujets du Sultan, notamment la judicature religieuse et le notariat musulman. En cela, il est pris à son propre piège : les autorités coloniales ont fabriqué une coutume qui justifie son

\footnotetext{
${ }^{7}$ Le cadre légal, que nous détaillons plus bas, prévoit un dédommagement aussi bien pour les militaires euxmêmes que pour les ayants droits (femmes et enfants) en cas de décès du soldat ou en cas de blessure. Cette question d'ensemble est très bien connue grâce à la thèse d'Antoine Prost, Les Anciens Combattants et la société française (1914-1939), 3 vol., Paris, Presses de la FNSP, 1977.

${ }^{8}$ Beaucoup de monographies consacrées à Lyautey ne manquent pas d'esprit critique mais n'ont pas toujours les moyens de comparer la lettre et l'esprit de la politique du maréchal. Ce faisant, ils ont tendance à prendre ce que dit Lyautey pour argent comptant, par exemple sur son respect d'une insondable «identité marocaine » (par exemple Robin Bidwell, Morocco under Colonial Rule, French Administration of Tribal Areas 1912-1956, London, Frank Cass, 1973, p. 18). L'empreinte de Lyautey est également très forte dans l'étude de ses successeurs : William A. Hoisington, The Casablanca Connection. French Colonial Policy, 1936-1943, London, The University of North Carolina Press, 1984.
} 
immobilisme en général mais qui, dans ce cas précis, devient un obstacle. La Résidence générale fait alors penser, dans ce contraste criant entre la concorde affichée durant la Première Guerre mondiale et la gestion de ses conséquences, au "génie de la famille » des Guermantes. Ceux-ci, raconte Proust, professent partout leur dédain du rang aristocratique et leur amour de l'intelligence, comme le Protectorat proclame son attachement aux institutions musulmanes et sa reconnaissance envers les anciens combattants marocains. Pourtant, il existe chez les Guermantes un génie de la famille qui, derrière les intentions verbales, ramène toujours ces grands seigneurs à leurs habitudes aristocratiques pourtant dédaignées : nous assistons durant la politique des pensions à l'empire du même mauvais génie qui, malgré une reconnaissance affichée, rapporte la puissance coloniale à sa morgue envers la monarchie et à une relative impuissance.

Après avoir montré les effets de la scénographie lyautéenne de collaboration durant la Grande Guerre et les revendications bien réelles qu'elle suscite parmi les anciens combattants, il faut envisager les obstacles que comportent la coutume musulmane et les institutions religieuses selon les autorités coloniales pour reconstituer le blocage de la distribution des pensions.

\section{I - La chorégraphie lyautéenne de collaboration autour de la Première guerre mondiale}

La Première Guerre mondiale engage le Maroc, toujours maintenu comme État de droit international, aux côtés de la France sur le front ennemi comme deux alliés ${ }^{9}$. Cette image est d'abord destinée à la métropole. Le général Lyautey tient à maintenir, contrairement à la Tunisie $^{10}$, la fiction de la souveraineté, ce qui a pour conséquence d'engager les troupes chérifiennes dans un corps spécial inassimilable à l'armée métropolitaine. Il affirme à nouveau le caractère permanent d'un régime qu'il estime plus accompli qu'en Tunisie, thème habituel

\footnotetext{
${ }^{9}$ Ces renseignements sont tirés de Mbark Wanaïm, «Le régime administratif des unités marocaines entre la première et la seconde guerre », Cahiers de la Méditerranée, n 78, 2009, p. 317-334. Voir aussi Moshe Gershovich, «Memory and Représentation of War and Violence : Moroccan Combatants in French Uniforms during the Second World War », in Ali Al Tuma, Eric Storm, Colonial Soldiers in Europe, 1914-1945, Aliens in Uniform in Wartime Societies, New York, Routledge, 2015, p. 78.

${ }^{10}$ Placée elle aussi sous Protectorat depuis 1881-1883 mais dont les troupes sont, comme en Algérie, soumises à la conscription. Les dossiers relatifs à leurs pensions, conservés au Service historique de la Défense, n'ont pas fait l'objet d'un dépouillement semblable dans le cadre de notre thèse de doctorat pourtant comparative.
} 
du Résident ${ }^{11}$. Il s'en justifie dans les termes suivants :

Pour ce qui concerne le recrutement des indigènes au Maroc, je ferai observer, en premier lieu, qu'il s'agit, non seulement d'un pays de Protectorat, mais d'un Protectorat d'une nature toute spéciale et, on peut le dire, sans similaire, puisque, plus encore que la Tunisie, le Maroc a été maintenu par les traités internationaux dans la situation d'un État qui, bien que protégé, jouit d'une réelle autonomie, sous la souveraineté effective du Sultan, non seulement Chef politique, mais encore Chef religieux. ${ }^{12}$

L'argument de Lyautey a deux aspects, l'un diplomatique, puisqu'il renvoie aux traités internationaux, et l'autre que l'on pourrait dire pittoresque, puisqu'il justifie l'indépendance $\mathrm{du}$ Maroc par la stature de son souverain, Commandeur des croyants. Le recours aux conventions internationales est peu convaincant : le fractionnement en deux actes (le traité du Bardo et la convention de la Marsa) de l'établissement du Protectorat tunisien en comparaison de l'unique traité de Fès ne suffit pas à justifier une différence qui, sur le plan du droit international, n'existe nulle part. Les deux États ont fictivement consenti à la même cession de souveraineté, et celle du bey n'est pas moins intouchée, en pure théorie, que celle du Sultan ${ }^{13}$. C'est le deuxième argument qui est plus significatif : c'est le charisme de la figure séculaire du Amīr al-mu'minīn ${ }^{14}$ qui a permis à Lyautey de consolider l'indépendance politique du Maroc et de sa propre marge de manœuvre ${ }^{15}$.

Le Sultan est, dans cet ordre d'idée, mis à contribution pour réaffirmer l'union indéfectible des deux États à l'occasion du conflit international. Moulay Youssef s'adresse aux troupes marocaines mobilisées en France, amenées à défendre la puissance protectrice injustement «agressée par l'État allemand», la métropole ne faisant que «défendre l'honneur de sa nation $»^{16}$. Et s'il appelle ses soldats à leur ancien « courage » (shuja 'an), c'est au nom d'une solidarité nouvelle entre les deux pays. Son argument est plus fort encore, il vise à décourager toute indifférence pour l'honneur d'une patrie étrangère :

\footnotetext{
11 « Nous ne sommes pas en Tunisie (...) là-bas, le Protectorat est une fiction ; ici il doit être une réalité » écrit Lyautey au ministre des Affaires étrangères le 13 août 1913, Gilles Ferragu, Lyautey, la fabrique du héros colonial, Paris, Belin, 2014, p. 143.

12 Mbark Wanaïm, art.cit.

${ }^{13}$ Pour un point de vue sur la doctrine du Protectorat, voir Annie Déperchin et Farid Lekeal, « Le protectorat, alternative à la colonie ou modalité de la colonisation? Pistes de recherche pour l'histoire du droit», Clio@Themis, Revue électronique d'histoire du droit $\mathrm{n}^{\circ} 4,2011$.

${ }_{14}$ «Commandeur des croyants », titre traditionnel des califes, successeurs du Prophète, chefs de la communauté spirituelle.

${ }^{15}$ Ce thème important sert également de contre-propagande à l'appel au Jihād du Sultan ottoman : cet épisode de la Grande Guerre mérite encore, comme le souligne Odile Moreau, beaucoup d'enquêtes. Odile Moreau, «Le Maghreb : un front oublié de la Première Guerre mondiale ? », Hespéris-Tamuda, LIII (1), 2018, p. 11-24.

16 «al-mudāfi' 'an al-sharaf wațanahā» Le Sultan Moulay Youssef aux combattants marocains en France, 19 août 1914, dans Muhammad Nahlīl, Rasā’il sharifiyya [Lettres chérifiennes], introduction et présentation AlJilālī Al-'Adnānī et 'Abd al-Raḥīm b. Hāāda, Rabat, Faculté de Lettres et Sciences Humaines, 2013 (1912), p. 245.
} 
Aucun ne vous ne pourrait douter que l'État français est venu au Maroc afin de prendre un soin particulier à l'amélioration de sa situation (biqașad mudīd al-i 'āna fí taḥsinn aḥwālaha), d'y organiser son œuvre (tanzīm a'mālaha) pour rendre cette région meilleure. Toutes affaires de l'État français constituent, avec le Maroc, une cause unique (șāra ma 'a al-dawla al-mag̀ribiyya kul shay' al-wāhid). ${ }^{17}$

Le Protectorat a alors instauré, dans la bouche même du souverain, une union des cœurs entre les deux États. Elle est de nature à justifier le sacrifice de vie de Marocains pour le profit de la France qui se confond avec celui du Maroc. Ce thème de concorde guerrière se répand dans tout l'empire chérifien. Des souscriptions sont organisées dans les villes pour les soldats marocains partis combattre au front. On en trouve un exemple dans les fonds privés du Pacha Șbīhī, gouverneur de Salé, qui préside une commission de bienfaisance (al-jana al-khayriyya) organisant une souscription pour les soldats partis en France. Dans un courrier écrit en arabe dans un style makhzen parfait, la femme du général Lyautey prête son concours aux desseins de communication de son mari. Elle remercie en 1915 les « habitants de votre ville pour leurs beaux sentiments pour leurs frères marocains, héros partis armés de leur courage dans les rangs alliés $»^{18}$. Muhammad al-Ṭayyib Ṣbīhī, aurait récolté auprès de la population plus de 122000 basìt ăt makhzaniyyăt $t^{19}$ que le chef des services municipaux lui a remis et qui seront versés à la Croix-rouge ${ }^{20}$. La guerre est une des occasions d'affirmer la bonne entente, en tout cas sur cette matière, des élites au niveau du Palais comme à l'échelle locale.

Après la bataille, les discours des autorités françaises réaffirmant la grandeur de la lutte commune ne manquent pas : le gouvernement français et les autorités de la Résidence tentent d'exploiter au mieux cette guerre au profit d'un thème de propagande, l'entente francomarocaine. Plus encore, il semble que les autorités coloniales voient dans cette expérience réussie de collaboration l'occasion de renforcer le Protectorat comme régime consenti par le Sultan, le Makhzen et, par conséquent, les Marocains. C'est pourquoi, en juillet 1919, le ministre de la Guerre convie les «hauts personnages marocains", avec les autres «grands chefs indigènes des pays d'Afrique du Nord » pour une célébration collective à Paris, pour donner à ces hommes d'importance « l'impression durable et féconde de la grandeur de notre Victoire $»^{21}$. Le général Gouraud fait bonne exécution dans le cadre informel d'un toast, après une visite de l'ossuaire de Verdun et une visite de la Galerie des Glaces de Versailles, où il

\footnotetext{
${ }^{17}$ Ibid. Littéralement : «Et toutes choses sont devenues, avec l'État marocain, une seule. »

18 «ahl madīnatikum min al-'awātîf al-jamīla nahū ikhwānikum al-maḡāriba al-abțal al-musā'irīn bikuli shajā‘a fì juyūsh al-duwal al-mutahālifa » Khizāna ṣbīhiyya, Bibliothèque Șbīhị de Salé, madame Lyautey au Pacha Șbīị̄i, 11 juin 1915.

${ }^{19}$ Monnaie du Maroc avant le Protectorat avant sa conversion au franc.

${ }^{20}$ Khizāna ṣbīhịiyya, Bibliothèque Șbīhī de Salé, madame Lyautey au Pacha Ṣbīḥī, 11 juin 1915.

${ }^{21}$ AMAE, 73CPCOM/17, Note du ministère de la Guerre, 7 juillet 1919.
} 
prononce les mots suivants :

Messieurs, il faut reconnaître que, si la France par sa justice, son libéralisme, sa sollicitude, par la prospérité toujours croissante, qu'elle a su développer dans ces Pays de l'Afrique du Nord qu'Allah lui a confiés, si la France, dis-je, a mérité que ses fils d'adoption la défendent au jour du danger, il faut reconnaître qu'elle a été généreusement récompensée par leur fidélité et leur courage. ${ }^{22}$

Le général insiste sur une concorde qui excède de très loin les modalités initiales du Protectorat comme forme juridique. 1918 est bien le moment où les autorités coloniales pensent utile, en s'appuyant sur la collaboration non plus seulement de l'élite palatiale mais aussi de simples soldats, de réaffirmer sinon d'intensifier les liens établis avec le Maroc et la Tunisie par les traités antérieurs. Reprenant les thèmes et le langage, jugé par tout l'état-major admirables, de Lyautey, il lève encore son verre au dessert :

Je lève mon verre en l'honneur de Sa Majesté Moulay Youssef, qu'Allah continue à lui accorder la santé et le bonheur dans son empire comme dans sa famille, de Son Altesse le Bey Si Mohamed Ben Naceur, qu'Allah lui accorde comme à Sidna la prospérité, de Monsieur le général Lyautey, à qui vont partout où quelques Marocains sont réunis, le tribut invariable de reconnaissance d'admiration et de fidélité. ${ }^{23}$

Maniant les formules et les bénédictions classiques du discours arabe, poussant la complaisance à appeler, comme Lyautey, le Sultan Sidna (notre seigneur) ${ }^{24}$, le général convie un thème qui reçoit un certain écho auprès d'une partie des élites marocaines qui manifestent leur enthousiasme. Une lettre, rédigée dans la perfection ornementale du style Makhzen, par 'Abd Allah al-Fāsī au ministre des Affaires étrangères, montre toute la reconnaissance d'un ami de la France. Ambassadeur à Paris avant le Protectorat, grand vizir du Sultan Moulay 'Abd al-ḥafîd, c'est en son nom personnel qu'il écrit au ministre ${ }^{25}$. La qualité dont il se réclame de représentant du Sultan à Fès (nẩib al-sultạn fì-l-madīna făs) semble plutôt honorifique. Il adresse ses « félicitations pour le règlement du conflit selon le désir de votre État glorieux (dawlatukum al-fakhīma)». Le grand vizir du Maroc, Muḥammad al-Muqrī, manifeste la même reconnaissance pour la tranquillité réservée au Maroc par Lyautey à l'occasion de son départ en 1924, car tandis que les autres États étaient en proie au grand conflit ravageur,

Le territoire marocain fortuné (al-quṭ al-maġribī al-sa ‘idd), par la grâce de votre autorité et par celle de

\footnotetext{
${ }^{22}$ AMAE, 73CPCOM/17, discours du général Gouraud du 23 juillet 1919. ${ }_{23}$ Ibid.

${ }^{24}$ Il ne manquait pas une occasion de se présenter comme le "premier serviteur de sidna" comme le relate Charles-André Julien, L'Afrique du Nord en marche, Paris, Omnibus, 1982, p. 51.

${ }^{25}$ AMAE, 73CPCOM/17, 'Abd Allah al-Fāsī au ministre des Affaires étrangères, 30 juin 1919. Voir sur ce personnage le livre de documents et de témoignages édités par son petit-fils Mohammed Fassi-Fihri, Mon grandpère, ambassadeur à Paris. 1909-1910, Rabat, Marsam, 2008.
} 
Sa Majesté le tout puissant roi [le Sultan] est resté dans sa quiétude physique et morale (sukūn aladaliyya wa-l-mādiyya $)^{26}$

La Première Guerre mondiale donne ainsi l'impression du premier succès de la politique lyautéenne. Mais si le territoire est en effet tenu avec peu d'hommes durant le conflit, ses conséquences sont beaucoup moins maîtrisées par les autorités coloniales. Leurs difficultés, en plus de problématiques liées aux rapports parfois conflictuels entre Lyautey et la métropole, révèlent les contradictions du gouvernement du Maroc par deux États, la monarchie et l'État colonial, sous le Protectorat.

\section{II - L’amertume des soldats marocains de la Première Guerre mondiale}

Au-delà des félicitations de l'élite makhzénienne, les simples soldats vont très vite se montrer déçus par les suites du grand conflit mondial. Tout incite les Marocains à attendre de la France une récompense à la hauteur de leurs efforts. Du point de vue du gouvernement français, la particularité du Protectorat ne résiste pas à l'exceptionnalité de la situation militaire: les Marocains obtiennent droit à la reconnaissance morale que l'État français accorde à ses citoyens ${ }^{27}$. C'est pourquoi les deux lois de 1919 et 1924 n'entendent pas exclure les troupes dites coloniales qui sont alors emportées dans le mouvement général de «l'économie morale de la reconnaissance $»^{28}$. La loi du 14 avril 1924, complétant celle sur les pensions militaires du 31 mars 1919, intègre elle-aussi les soldats non-citoyens d'Afrique du Nord dans le régime des pensions, le législateur « estimant sans doute qu'en combattant pour la France, ils avaient contribué à son salut et l'avaient momentanément au moins considéré comme leur patrie adoptive $»^{29}$. Le Sultan lui-même, dans son épître aux soldats partis combattre en France, n'avait pas dit autre chose :

Vous gagnerez un honneur (sharaf) dont hériteront vos fils génération après génération (jīlān ba'd jül) (...)

Et dans une année proche, vous reviendrez victorieux (zâfirīn) dans notre noble terre

\footnotetext{
${ }^{26}$ CADN, 1/MA/300/136, Le grand vizir au Maréchal Lyautey, s.d. (c. 1925).

27 Sur ce lien paradoxal avec la métropole, à la fois renforcé et affaibli par la recomposition des hiérarchies raciales, voir l'introduction de Ali Al Tuma dans Eric Storm (dir.), op.cit., qui souligne la relative discrétion historiographique de cette question.

${ }^{28}$ L'expression est utilisée par Bruno Cabanes, La victoire endeuillée : la sortie de guerre des soldats français : 1918-1920, Paris, Seuil, 2004.

29 Jean Thomas, «La loi sur les pensions militaires dans son application aux musulmans indigènes non naturalisés de l'Algérie, de la Tunisie et du Maroc », Revue algérienne, marocaine et tunisienne de législation et de jurisprudence, mars-avril et mai-juin 1921, p. 41-91, p. 7.
} 
qui est aussi la vôtre, et vous obtiendrez, comme fruits de votre travail (natijja 'amalikum), l'assurance d'un avenir heureux (sa 'âda al-mustaqbal). Vous obtiendrez pour votre service et votre devoir accomplis la meilleure des récompenses (ậsan al$m u j \bar{a} z \bar{a} a)$, et vous pourrez louer la grâce de Dieu $(h \bar{u} l \text { Allah })^{30}$.

Naturellement, le Sultan reste imprécis sur la nature de cette récompense, mais il laisse supposer qu'elle sera moins immatérielle et abstraite que le simple honneur : l'assurance d'un avenir heureux est une préoccupation bien terrestre, pour laquelle ils pourront remercier le Sultan. Depuis Moulay 'Abd al-raḥmān (1822-1859), l'existence d'une armée permanente (jaysh nizāmi $)$ garantit des soldes régulières aux soldats qui pouvaient combattre autrefois sans rétribution ${ }^{31}$, elle est calculée de façon à pourvoir aux besoins vitaux et familiaux des soldats, à l'inverse de bien des traitements du Makhzen chérifien plus honorifiques qu'alimentaires $^{32}$. L'idée d'un «traitement» (rātib) s'est donc imposée avant l'arrivée de la France aux militaires marocains. La cohabitation avec les soldats français au front pouvait leur inspirer l'idée même de pension qui se confond alors dans leur esprit avec la solde ou le salaire du soldat ${ }^{33}$. Après la guerre, la différence de traitement entre Français et Marocains est constatée par les anciens combattants marocains : le contrôleur civil de Mogador note en 1924 que les mutilés marocains remarquent que «les blessés français avaient le droit d'être convenablement assistés par la nation» et raconte au Résident général l'amertume de «manchots, unijambistes, tuberculeux, vêtus de haillons, faméliques » qui déplorent l'injustice de leur sort ${ }^{34}$. Le commandement militaire s'est, sans doute, lui-même fait le reflet de cette propagande de la récompense au sein des bataillons, pour animer leur courage ou leur adhésion.

À la fin des années 1920, la préoccupation des autorités du Protectorat augmente face à une rumeur qui court, dans les «milieux indigènes », d'une prochaine cessation de paiement

\footnotetext{
${ }^{30}$ Le Sultan Moulay Youssef aux combattants marocains en France, 19 août 1914, in Muhammad Nahlīl, op.cit, p. 245.

${ }^{31}$ Turayyā Barrāda, Al-jaysh al-magribī wa tațawuruhu fí-l-qarn al-tāsi' 'ashr [L'armée marocaine et son évolution au XIXe siècle ], Rabat, Maktaba Ibn Khaldūn, 1997, p. 231.

${ }^{32}$ Ainsi l'explique Mușțafā Al-Shābī, spécialiste de l'élite makhzénienne, Muștafa Al-Shābī, Al-Jaysh al-mag̉ribī fì-l-qarn al-tāsi' 'ashr 1830-1912 [L'armée marocaine au XIX ${ }^{e}$ siècle], Rabat, al-muṭba'a wa-l-warāqa almagiribiyya, 2008. Il montre en réalité une forme très encadrée de traitement en dehors des variations habituelles au Makhzen de l'éminence familiale, des rapports personnels (p. 318). Il reste des imperfections dans le versement de ces soldes, dues aux difficultés financières et les salaires se montrent vite insuffisants pour les soldats, ils restent stagnants sur toute la période (p. 332-338).

${ }^{33}$ La bibliographie ne fait pas mention d'une «pension » au XIX ${ }^{\mathrm{e}}$ siècle, au sens où des dispositions spéciales pour les blessés ou les familles. Celles-ci ne semblent pas exister de façon régulée mais, à charge de preuve du contraire, il est autorisé de penser que la générosité du Sultan pouvait s'étendre aux familles affligées selon des procédés plus invisibles qu'un texte de loi et par conséquent difficiles à restituer. Mușțafā Al-Shābī donne plusieurs exemples de sollicitude des autorités face aux afflictions des soldats (op.cit., p. 427 et suiv.).

${ }^{34} \mathrm{CADN}, 2 / \mathrm{MA} / 1 / 49$, Le Contrôleur civil de Mogador au Résident général, 7 août 1923.
} 
des pensions de retraite aux anciens militaires marocains, notamment à Marrakech et à Meknès, selon les autorités militaires ${ }^{35}$. Cette inquiétude serait un écho au mécontentement d'anciens militaires dont la pension aurait été retirée, après un changement de résidence des pensionnés sans avertissement à l'autorité, ou bien par défaut du service des pensions qui ne dispose que d'adresses incomplètes. La «complexité des formalités administratives ${ }^{\mathbf{3 6}} \mathrm{se}$ heurte à toute une série d'ignorances et d'absence d'institutions compétentes. Deux des rares manuscrits arabes d'anciens soldats sur cette question militaire peuvent l'illustrer. Ils mettent en scène une inquiétude et un mécontentement que les sources militaires estiment généralisées.

La compréhension de ces deux manuscrits, écrits dans une graphie hésitante, est rendue difficile par le mélange entre le dialecte marocain et l'arabe littéraire. Par exemple, la translittération du mot « France », dans les deux cas, ne tient pas compte de la translittération normée de l'arabe écrit (franș ou frānsīs au lieu de faransā). Il témoigne surtout d'une absence de maîtrise de la langue savante et d'une origine sociale populaire, qui devait être la règle pour les soldats du contingent marocain. Celle-ci éclaire, d'une certaine façon, l'aspect «bénévole » de la mobilisation de soldats qui étaient des sujets marocains dans une situation économique précaire. Deux situations peuvent être mises en comparaison dans le début des années 1920 : Idrīs b. Muhammad se présente comme venant du 6e régiment, ayant combattu en France il a gagné une médaille militaire ${ }^{37}$. L'autre se présente comme 'Ayād b. Bashīr, de la tribu des Awlād b. Hamdūn, son fils Mahdī est mort en France au combat. Les deux demandent explicitement une subvention, soit à titre personnel, soit à titre familial. Le père endeuillé voulait (bgit) des renseignements et il s'est fait répondre qu'il n'existait pas d'allocation (si 'anwa renvoie à l'arabe classique $i$ 'āna). Quant à Idrīs b. Muhammad, il raconte que le bureau militaire lui a dit qu'il n'aurait pas de « retraite » (ntarît, désignant la pension ${ }^{38}$ ) et qu'on ne « lui donnerait pas d'argent» (lā ya 'ṭānī flūs) pour sa médaille militaire, alors même qu'il n'a « aucun endroit pour vivre» (ma'andīsh blāsā bāsh an 'issh). Il se trouve enfin dans un état très misérable (faqīr $d a$ ' $\imath f$ ). Ces deux courriers obéissent à la tradition des requêtes ou shikāyāt présente au Maroc comme dans le reste du monde musulman: les sujets peuvent

\footnotetext{
${ }^{35}$ CADN, 1/MA/200/484, Le Commandant de la Région de Meknès au Résident général, 20 juin 1929.

${ }^{36}$ CADN, 1/MA/200/484, Le chef du service du contrôle civil au Directeur général des affaires indigènes, 22 juillet 1929.

${ }^{37}$ CADN, 1/MA/100/252, Idrīs Ibn Muhammad au Résident général, 2 septembre 1921.

${ }^{38}$ Nous constatons en effet en d'autres endroits que si la retraite comme état de la vie après le travail se rend bien par taqā'ud, l'idée de pension de retraite vient du français intirāt. Voir à titre d'exemple le cas d'un Amīn des Ports qui distingue ces deux notions dans son courrier du 20 février 1934 au Résident général (CADN, 1/MA/100/399).
} 
s'adresser au prince qui, détenteur de la justice de redressement des torts (mazāalim), peut corriger une injustice ${ }^{39}$. Mais, sous le Protectorat, le Résident s'est substitué au Sultan. L'amertume exprimée par ces lettres est manifestement partagée par bien d'autres anciens combattants qui n'ont pas les ressources pour écrire au pouvoir central. Du reste, Idrīs b. Muhammad évoque ses «pairs militaires» (widāanā al-'askrî̀) qui sont dans la même situation.

Plus essentiellement que la réclamation d'un droit, c'est la traduction des réalités bureaucratiques militaires dans le langage dialectal qui frappe ici. Ces deux demandes sont, par certains côtés, très précises (le numéro d'immatriculation de soldat, le régiment) mais aussi très vagues sur la nature des récompenses auxquelles les Marocains peuvent prétendre. Surtout, c'est la nature sémiotique des noms mêmes des institutions qui frappent: les deux évoquent un « bìru » pour désigner l'endroit où ils se sont rendus. De la même façon, la « médaille militaire » (midāa mlītìr) est translittérée directement en arabe, comme la pension qui est assimilée à une retraite. La présence du français en graphie arabe signifie que l'État n'a produit aucune administration arabisante d'interface avec les Marocains. Ceux-ci doivent intégrer des mots, sans analogie avec des institutions traditionnelles ou du moins sans attribution d'un mot équivalent en arabe. Le rôle habituel des Umanā ${ }^{‘ 40}$, chargés de distribuer les soldes sous la direction du 'Allāf al-kabīr, ministre de la guerre, a été visiblement évincé ${ }^{41}$. Les soldats en ont pris conscience et montrent, dans une langue inventive qui intègre au XIX siècle de nombreux mots d'origine étrangère, le face-à-face avec des institutions françaises de la puissance coloniale. Un potentiel malentendu apparait : incapables de formuler dans un langage juridique leurs revendications par des références précises, les deux plaignants voient la porte de la nation reconnaissante se fermer devant eux. La légèreté des procédés du bureau militaire est ici aussi frappante que l'imprécision technique des deux Marocains : au père de Mahdī on donna « dix riyals » en promettant que le gouverneur (hakām) agirait (dirt) pour

\footnotetext{
${ }^{39}$ La bibliographie est étendue sur ce sujet, voir à titre d'exemple Yadh Ben Achour « Justice des Madhalim et justice administrative moderne », Revue internationale des sciences administratives, Bruxelles, 1985, $\mathrm{n}^{\circ} 2, \mathrm{p}$. 109-115 et pour un rapport critique à celle-ci Mathieu Tillier, "The Mazālim in Historiography », in A.M. Emon et R. Ahmed (éd.), The Oxford Handbook of Islamic Law, Oxford, Oxford University Press, 2015 [en ligne].

${ }^{40}$ Forme d'intendants chargés de mission économiques auprès des postes, des ports, des marches, de la douane, corps créé à la fin du XIX ${ }^{\mathrm{e}}$ siècle par Moulay Hassan. A ce sujet : Na'īma Harāj Al-Tūzān̄̄, Al-Umanā' bi-lmag̉rib fì 'ahd al-sulțān Mawlāy Hasan [Les Oumanas au Maroc à l'époque du Sultan Moulay Hassan], Rabat, Université Mohammed V, 1979.

${ }^{41}$ Bahija Simou montre la dimension réglée et fixe des soldes délivrées par un amīn, sous la surveillance de différents registres et autorités attestant des droits des pensionnaires. Le amīn effectue les paiements en présence de la hiérarchie militaire qui en vérifie l'exactitude. Bahija Simou, Les réformes militaires au Maroc de 1844 à 1912, Rabat, Université Mohammed V, 1995.
} 
lui ${ }^{\mathbf{4 2}}$, et on ne livre aucun renseignement à Idrīs b. Muhammad. Le quiproquo semble donc complet entre des Marocains réclamant la gratitude de la France et des agents français incapables de leur répondre. La pension devient, au fil des années, un problème social.

La première ligne de l'État colonial au Maroc, les contrôleurs civils, soulignent alors la nécessité d'« étudier (pour les Marocains) une réglementation plus en rapport avec leur mentalité et leur ignorance de la législation $»^{43}$. Ignorance bien partagée en réalité, puisque les autorités de contrôle se plaignent elles-mêmes de ne pas toujours être mises au courant des nouvelles procédures ou de ne pas disposer d'imprimés idoines. Derrière ces problèmes pragmatiques se cachent des situations impossibles : l'administration ne sait pas où sont les pensionnés, ni comment les contacter, ou comment déterminer précisément leurs droits par rapport à cette politique métropolitaine.

Pourtant, les autorités militaires marquent une sollicitude véritable, tout en étant condescendante $^{44}$ envers les anciens militaires marocains : le commandant du centre spécial de Réforme ${ }^{45}$ affirme, face à une réclamation d'un ex-soldat du 2e régiment, que compte tenu du délai de prescription « il y a une importance absolument capitale à retrouver cet indigène, à établir son identité et à lui donner une pièce d'identité car il n'en possède aucune ${ }^{\mathbf{4 6}}{ }^{\text {. Ce sont, }}$ nous le verrons, les autorités de contrôle qui se montrent plus rétives et qui cherchent des solutions de mauvaise grâce, forcées progressivement par la situation. Les responsables militaires s'entendent en effet avec les autorités résidentielles sur «l'intérêt politique » de ces réformes. Le problème se politise finalement et s'étale sur plusieurs décennies : le 30 avril 1934, le président de la section des Anciens combattants républicains de Meknès écrit au Résident général ${ }^{47}$. Ce Français s'inquiète de la réduction de la pension des Marocains, par suite de politiques générales d'économie. L'argumentaire présente alors sous un jour rude la

\footnotetext{
${ }^{42}$ Dans les limites de nos compétences paléographiques, il semble que la traduction du Protectorat soit calomniatrice : le requérant aurait prétendu que le bureau lui a dit qu'on lui donnerait des renseignements moyennant cinquante francs, or le plaignant précise simplement « on m'a donné dix riyals » ( 'ațīn riyāla) et il n'en a point proposé.

${ }^{43}$ CADN, 2/MA/1/49, Circulaire du Secrétaire général aux contrôleurs civils, 5 mai 1924.

${ }_{44} \mathrm{CADN}, 2 / \mathrm{MA} / 1 / 49$, L'Intendance souligne la nécessité, auprès du Protectorat, de considérer les indigènes comme «des enfants mineurs vis-à-vis desquels l'autorité de contrôle, ou le Service de renseignement, rempliraient le rôle de parents ou de tuteurs » afin de garder leur papier, idée très peu au goût des services de contrôle. Note de l'Intendant général, 22 février 1923.

${ }^{45}$ Centres de démobilisation des soldats pendant et après les conflits, plus particulièrement chargés d'estimer les taux d'invalidité et donc les taux de pension.

${ }^{46} \mathrm{CADN}, 2 / \mathrm{MA} / 1 / 49$, Le médecin commandant chef du centre spécial de Réforme au chef du bureau des affaires indigènes, 22 mai 1933

${ }^{47}$ AMAE, 73CPCOM/349, Le président de la section des Anciens combattants républicains de Meknès, 30 avril 1934. La documentation fait un bon du milieu des années 1920 au milieu des années 1930 sans que nous puissions, pour l'instant, en remarquer la raison.
} 
situation des anciens pensionnés : la pension, "déjà infime », ne suffit pas en raison de l'augmentation du coût de la vie aux « malheureux indigènes dont beaucoup ne sont plus que des déchets humains, en raison des mutilations qu'ils ont subies au service de la France » et qui «ont été rejetés sur le pavé avec une pension insuffisante pour leur permettre de vivre ». Ces Français prennent fait et cause pour leurs "protégés », soudain unis à eux par une solidarité militaire qui s'impose au rapport de force colonial; comme en métropole, les mutilés marocains sont décrits comme des « déchets humains $»^{48}$. Mais, en dernière analyse, le rapport de force colonial revient comme une préoccupation : «Nous pensons, monsieur le Résident général, que la situation politique n'est pas, à l'heure actuelle, tellement brillante au Maroc pour qu'on puisse songer, par une mesure inopportune, à apporter à nos protégés un nouveau sujet de mécontentement ». Devant ce danger politique, la Résidence s'empare de la question et défend l'intérêt des pensionnés marocains auprès de la métropole, une fois n'est pas coutume. Mais, en cette matière, le poids pour le budget n'était pas trop important compte tenu du peu d'anciens combattants, ce qui permet de réconcilier, avec soulagement, la rétribution patriotique des indigènes et les exigences financières du Protectorat. Ce n'est pas tant sur le principe de cette politique publique que dans son application que l'écart se creuse entre les espoirs d'état-major et les pratiques de contrôle civil.

\section{III - Les obstacles d'une politique publique : les pensions et la « coutume musulmane »}

Sur un plan technique, le Protectorat doit trouver les moyens de liquider des pensions qui relèvent d'un argent métropolitain. Mais si l'argent vient de France, le Maroc reste souverain dans la gestion de son trésor, effet du Protectorat que rappelle le Conseil d'État ${ }^{49}$. La métropole insiste pour que le Maroc applique une législation française à l'image de la Tunisie et non, comme le suggérait la Direction des affaires civiles à Rabat, un règlement proprement

\footnotetext{
${ }^{48}$ Clément Collard, préparant une thèse de doctorat sur les mutilés de la Première mondiale, qu'il soit remercié pour cette référence, remarquait dans la bouche de J. Thibaudeau, directeur de l'école de rééducation professionnelle de Bordeaux dans les années 1930, ce mot d'ordre et cet impératif : "d'un "déchet humain" faire un travailleur, un producteur, un homme utile, quelle plus noble tâche ! », livret sur l'école publié en 1936, Archives Départementales d'Ille-et-Vilaine, cote 3X743.

${ }^{49}$ Arrêt du Conseil d'État du 16 décembre 1932, Recueil Lebon, Paris, Delhomme, 1932, p. 1090. L'arrêt précise que si la pension appartient au Trésor Public français, le traitement étant réglé par le Maroc, le directeur de la Dette inscrite au ministère des Finances ne peut donner d'ordre au Trésorier général du Maroc.
} 
marocain, pour des « raisons d'ordre politique et moral ${ }^{50}$. Ainsi, l'origine métropolitaine change la nature de cette politique publique : le Protectorat ne peut plus se contenter des auxiliaires habituels du Makhzen sultanien pour ses interactions administratives avec les Marocains, il doit distribuer directement ces pensions sans passer par ses intermédiaires coutumiers. Il se heurte alors à une ignorance commune à tout territoire de l'empire français, l'impossibilité de compter ses sujets ${ }^{51}$ : l'état-major entreprend des premiers comptages, ce qui porterait à 1780 un total d'anciens militaires pensionnés ou médaillés militaires, en $1923^{52}$. Mais jamais l'autorité militaire ne dispose de listes exhaustives, faute de pouvoir recenser les adresses de Marocains parfois nomades ${ }^{53}$. En fait, les sujets du Sultan semblent en grande partie inaccessibles aux autorités de Protectorat, sans secours du Makhzen pour sa connaissance de la société marocaine, pour ses réseaux d'agents, et enfin pour ses propres formes d'organisation administrative. La tentative de contournement du Makhzen par le Protectorat se traduit par la volonté d'enjamber plusieurs obstacles qui constituent le fond de la « coutume musulmane ».

\section{L'obstacle de la langue arabe}

La dimension hybride franco-marocaine de cette politique publique pose des problèmes spécifiques au contexte nord-africain. En janvier 1932, le chef de la circonscription de Zemmour signale le cas d'un pensionné indigène qui a signé son livret de pension en graphie arabe, d'une façon somme toute assez naturelle puisque c'est la langue de son nom, et non en alphabet latin translittéré54. Il l'a retourné à la Trésorerie de Rabat, qui lui a fait savoir que sur les «pièces de dépenses métropolitaines", la signature en arabe doit être certifiée par l'autorité dont le sujet protégé relève, c'est-à-dire un interprète assermenté du contrôle civil. L'interprète est un intermédiaire essentiel entre l'administration française et les Marocains, mais il est aussi coûteux pour des opérations qui doivent se répéter tous les trois mois pour la liquidation de la pension. Mais se passer d'intermédiaires reviendrait à faire déplacer les pensionnés à Rabat. Le cas de Zemmour finit par se régler finalement, le requérant ayant

\footnotetext{
50 À savoir «l'application des lois réparatrices des effets de la guerre ». AM, E0878, Note pour le Secrétaire général du Protectorat, septembre 1920.

${ }^{51}$ Raymond Gervais et Mandé Issiaka « Comment compter les sujets de l'Empire ? Les étapes d'une démographie impériale en AOF avant 1946 », Vingtième Siècle. Revue d'histoire, vol. 95, no. 3, 2007, p. 63-74.

52 CADN, 2/MA/1/49, le Chef du service des contrôles civils au Secrétaire général, 14 mai 1923.

53 CADN, 2/MA/1/49, Circulaire du Secrétaire général aux contrôleurs civils, 5 mai 1924.

$54 \mathrm{CADN}, 1 / \mathrm{MA} / 200 / 484$, Le Délégué à la Résidence générale à la Direction des Finances et à la Trésorerie générale, 28 janvier 1932.
} 
" appris à signer en français $»^{55}$, mais cette opération peut-être simple n'ôte rien, souligne le chef du contrôle civil, au caractère très lourd et contraignant de la procédure qui suscite un mécontentement légitime auprès de la population.

Ce problème est particulier à la politique des pensions : comme le souligne le directeur des Affaires indigènes au chef du service du Contrôle civil, les procédures sont différentes si les opérations se font au compte du budget chérifien ou si elles le sont au compte du budget métropolitain. Pour les premières, un acquit en langue arabe est admis, mais la signature en français est exigible pour le budget métropolitain, ainsi que le demande la Caisse des dépôts et consignations ${ }^{56}$. Le Protectorat, soucieux de s'épargner des besoins de traduction et d'inutiles lenteurs procédurières, a demandé à la Cour des comptes une modification des règlements de comptabilité publique. Mais si la métropole exerce une influence souvent irrésistible sur les procédures marocaines, l'inverse est rarement vrai : la Cour a refusé ${ }^{57}$, le Protectorat doit donc composer. Les autorités coloniales demandent à des sujets arabophones de signer en langue française, les forçant à accepter une assimilation linguistique et administrative puisqu'ils en réclament de l'argent, pour le dire d'une manière plus claire. Mais le problème est plus profond encore : les autorités de Protectorat se heurtent à l'impossible collaboration avec la forme d'état civil de la monarchie musulmane, la judicature religieuse. Ils doivent alors trouver des substituts qui coûteraient le moins cher possible, ce qui exclut beaucoup d'autorités françaises.

\section{Les 'udūl comme état-civil et la recherche de substituts}

L'acte d'importance permettant la distribution des pensions de réversion pour les ayants droits est l'attestation de mariage ou de filiation : or celui des sujets marocains est jugé d'un style trop particulier par les autorités du Protectorat. Ils sont délivrés par les cadis et ' $u d \bar{u} l^{58}$

55 CADN, 1/MA/200/484, Le chef du service du contrôle civil au Trésorier général du Protectorat, 23 février 1932.

${ }^{56}$ Archives diplomatiques (France, CADN), 1/MA/200/484, Le Directeur des Affaires indigènes au Chef du service du Contrôle civil, 19 avril 1934.

57 Ibid.

${ }^{58}$ Les cadis ( $\left.q \bar{a} d \bar{\imath} \mathrm{pl} . q u d \bar{a} t\right)$ sont les magistrats musulmans chargés de trancher, à l'époque coloniale, les litiges civils selon le fiqh (droit musulman) de l'école malékite. Les ' $u d \bar{u} l$ ('adl pl. 'udūl) sont les « notaires » (« adoul» dans la documentation coloniale) chargés d'enregistrer les actes et les témoignages. Pour un contrepoint tunisien : Hassan El-Annabi, «Les notaires musulmans de Tunisie », Cahiers de la Méditerranée, ${ }^{\circ}$ 63, 2001, $\mathrm{p}$. 41-51. 
qui peuvent être considérés comme les officiers d'état civil de la monarchie marocaine ${ }^{59}$. En conservant aux Marocains leur statut personnel, les autorités de Protectorat ont laissé aux soins de la judicature religieuse l'organisation de la vie des sujets protégés : actes de notoriété (naissance, décès, vie), mariage et succession dépendent d'une justice dite charaïque (al'adala al-shar 'ìyya), c'est-à-dire islamique, dont les deux figures essentielles sont les cadis et les ' $u d \bar{u} l$ d'institution très ancienne. À chaque juge religieux est adjoint deux ' $u d \bar{u} l$ d'où l'emploi systématique de la forme plurielle, les deux formant le tribunal en tant que tel, la $m a h k a m a^{60}$. Les actes de notoriété sont délivrés au sein des tribunaux religieux, où un des ' $u d \bar{u} l$ produit un document reconnu ensuite par le cadi. Le cadi et les 'udūl, nommés par le prince, participent à ce qu'il convient d'appeler une fonction publique musulmane, le cadi jugeant selon la majesté du droit religieux mais aussi au nom de l'État ${ }^{61}$. Les autorités coloniales, ne reconnaissant pas le témoignage comme une forme de preuve juridique, vont instaurer un état civil sur le modèle métropolitain pour les seuls Français, à Rabat puis, par le dahir du 18 juin 1913, à Kénitra et Mehdia afin de faciliter l'enregistrement des citoyens expatriés pour qui l'état civil est obligatoire. Il est facultatif pour les Marocains, sur le modèle du décret beylical tunisien du 29 juin 1886, pour ne pas provoquer des dépenses d'envergure. Le dahir du 4 septembre 1915 crée la possibilité mais non l'obligation d'un état civil chérifien, mais ce n'est qu'avec le dahir du 2 septembre 1931 qu'il rentre en réelle application pour les décès et les naissances, le mariage en restant exclu probablement pour ne pas intégrer des exemples de polygamie dans le droit $^{62}$. Les contrôleurs civils se substituent alors, en application du dahir, à la judicature religieuse, agissant en qualité de fonctionnaires chérifiens.

Dans l'esprit des autorités coloniales, il est ainsi impensable que les 'udūl soient considérés comme des officiers d'état civil. Cette tâche ne peut appartenir qu'à un agent français. Une note du Protectorat renseigne la procédure coutumière jugée inapplicable : les actes de notoriété, établissant les filiations, sont certifiés par « douze témoins honorablement

\footnotetext{
${ }^{59}$ Sabine Filizzola, L'organisation de l'état civil au Maroc, Paris, Librairie générale de droit et de jurisprudence, 1958, p. 17.

${ }^{60} \mathrm{La}$ science orientaliste a tôt fait d'expliquer ces institutions de la juridiction religieuse, à l'exemple de RenéJules Frisch, Henri David, Guide pratique en pays arabe, Paris, Berger-Levraut, 1892, p. 10.

${ }^{61}$ La nomination des cadis par le Sultan, traditionnelle (on en trouve un exemple dans le dahir de nomination de Aḥmad b. al-Buqqāl al-Warīshī, 12 mai 1889, Al-Watā'iq, nº18, 2013) est toujours d'usage sous le Protectorat. Dans un dahir, le maître califal délivre une partie de son autorité dans la judicature du cadi, ce qui l'introduit dans l'Etat : " Nous l'investissons solennellement dans ses fonctions juridictionnelles et l'autorisons à connaître les conflits et litiges entre les plaignants, qu'il jugera selon l'école de notre Imām Mālik ». («walīnā wa qaladnāhu bi-șafatiha al-ḥakmiyya wa aḍanā lihi fī tașfị̆ al-rusūm wa-l-faṣl bayn al-khușüm wa-l-ḥukm bishuhur madhab imāminā Mālik»).

${ }^{62}$ Sabine Filizzola, op.cit, p. 42.
} 
connus », ce qui crée des longueurs aux yeux des autorités coloniales ${ }^{63}$. L'honorabilité est en effet associée, en droit musulman, à la valeur du témoignage sur lequel se fonde le droit, c'est un principe élémentaire du fiqh ${ }^{64}$. Le notaire musulman est donc un peu plus qu'un fonctionnaire de bureau puisqu'il est augmenté du pouvoir de sa parole. Ceci sur un plan théorique, il existe toute une production écrite qui accompagne toute procédure du tribunal comme les sijīlāt ottomanes ${ }^{65}$.

Le problème tient plutôt à l'absence du contrôle, de la part de l'État colonial, des modalités d'administration de l'acte : celui-ci est rémunéré par les sujets marocains aux 'udūl, selon des prix qui varient d'une région à l'autre et des procédures déterminées par la liberté des secrétaires $^{66}$. En raison de l'hétérogénéité des tarifs (dont vivent généralement les 'udūl), les Marocains les plus pauvres sont lésés selon les autorités militaires, limite qu'elles attribuent à la nature de l'état civil musulman. C'est pourquoi le directeur de l'Office marocain des Mutilés, combattants et victimes de la guerre estime qu'on pourrait faire établir ces actes administratifs à titre gratuit par les services de contrôle qui vont vite s'en défendre ${ }^{67}$. Les autorités de Protectorat, soucieuses de ne pas faire intervenir la justice religieuse dans des matières purement françaises, souhaiteraient donc créer une sorte d'exception dans ce « monopole adoulaire » et consacrer une procédure unique. Elles ne peuvent pas collaborer avec les ' $u d \bar{u} l$ qui sont exclus, par une pratique tarifaire jugée archaïque, d'une procédure d'État voulue moderne. Mais il faut alors lui trouver des remplaçants.

L'hypothèse de confier cette tâche aux contrôleurs civils est exclue pour des motifs financiers. Le chef des contrôles civils déplore l'insuffisance du personnel ne serait-ce que

\footnotetext{
${ }^{63}$ CADN, 1/MA/200/484, Note non datée sur l'établissement des actes de notoriété pour les Marocains (mais probablement contemporaine des documents qui l'accompagne, vers 1938).

${ }^{64}$ L'importance du témoignage se lit dès le Coran en réalité où la preuve est associée au témoignage comme du reste dans la Bible. La primauté de la preuve (hujja) testimoniale est établie parmi d'autres dans un système complexe et variable selon les écoles. La «preuve par excellence » est le témoignage shahāda selon certaines conditions légales mais on peut lui opposer le serment (yamīn). Sur ces questions complexes voir Robert Brunschvig, «Le système de la preuve en droit musulman », Étude d'islamologie, Paris, Maisonneuve et Larose, $1976 »$, p. 209 s.q.

${ }^{65}$ Cette image figée de l'islam comme «tradition orale» a été démentie depuis longtemps par des travaux comme ceux de Gregor Schoeler, Écrire et transmettre dans les débuts de l'Islam, Paris, PUF, 2002. La procédure écrite du notariat musulman est surtout connue pour l'empire ottoman : Leslie Peirce, Morality Tales : Law and Gender in the Ottoman Court of Aintab, Berkeley, University of California Press, 2003 et Ronald J. Jennings, « Kadi, Court and Legal Procedure in 17th Century Ottoman Kayseri », Studia Islamica 48, 1978 , p. $133-172$.

${ }^{66}$ Nous rappelons que les contrôleurs civils sont chargés de «surveiller » en théorie les caïds et pachas, en inspirant en réalité la plupart de leurs actions. Le Maroc est divisé en zone militaire, où les contrôleurs sont des officiels, et zone civile le temps de la pacification. CADN, 1/MA/200/484, Circulaire aux contrôleurs civils du Directeur des affaires politiques, 8 août 1938.

${ }^{67} \mathrm{CADN}, 1 / \mathrm{MA} / 200 / 484$, Le Directeur de l'Office marocain des Mutilés combattants et victimes de la guerre au Directeur des Affaires politiques, 15 octobre 1936.
} 
pour conserver les papiers des anciens militaires marocains, d'où l'intérêt de les confier aux autorités du Makhzen ${ }^{68}$. Le Protectorat se résout alors à donner cette tâche aux pachas et caïds, autorités marocaines plus contrôlées que les magistrats religieux. Il faut attendre le dahir du 19 janvier 1938 pour que la mission d'établir les actes pour les ayant-droits des militaires et anciens militaires des pensions soit donnée aux pachas et caïds officiellement ${ }^{69}$, fonction déjà remplie par eux en pratique ${ }^{70}$. Le Protectorat a choisi un régime d'exception en confiant aux officiers makhzéniens ce qui devait échoir à l'État colonial en temps normal.

\section{L'obstacle construit des " usages indigènes "}

Cette exception ne doit pas se généraliser : étendre l'état civil à tous les Marocains, selon un contrôleur, «heurterait les coutumes de nos protégés qui ne paraissent pas encore suffisamment évolués sur ce point $\gg^{71}$. Les blocages de la Résidence sont visibles : elle a certes besoin du Makhzen comme médiation avec la population marocaine, mais le Makhzen ne fonctionne pas comme l'État colonial car il est esclave d'une insondable «tradition ». En réalité, l'usage de la notion de coutume est généralisé à tout l'empire français dans le droit des pensions. Le Conseil d'État en précise les principes, dans un avis rendu le 9 mai 1922, sur sollicitation du ministre de la Guerre et des Pensions, à propos du droit à pension des veuves et les ascendants musulmans ${ }^{72}$. L'article 74 de la loi du 31 mars 1919, portant sur les « indigènes musulmans non naturalisés originaires d'Algérie, de Tunisie ou du Maroc » pose problème au ministère : il prévoit l'allocation d'une pension à la «famille du militaire indigène », équivalente à la pension pour les veuves et les orphelins des militaires français. Pour ceux-ci, cette pension est non seulement partagée entre ces derniers, mais également avec les « éventuels ascendants d'après la décision rendue par l'autorité locale en s'inspirant des usages indigènes $»^{73}$. La section des Finances, de la Marine et des Colonies constate que les ascendants n'ont pas de droit absolu en soi, et que c'est au ministère de la Guerre d'en déterminer les conditions d'ouverture. Par conséquent, elle répond à une question importante :

\footnotetext{
${ }^{68} \mathrm{CADN}, 1 / \mathrm{MA} / 200 / 485$, Le chef du service des contrôles civils pour le chef d'état-major du Maréchal commandant en chef, 19 mai 1922.

${ }^{69} \mathrm{CADN}, 1 / \mathrm{MA} / 200 / 484$, Note non datée sur l'établissement des actes de notoriété pour les Marocains.

${ }^{70}$ Ils examinaient les dossiers individuellement dans des commissions. CADN, 2/MA/1/49, le Secrétaire général aux Directeurs et chefs d'administration, 8 septembre 1925.

${ }^{71} \mathrm{CADN}, 1 / \mathrm{MA} / 200 / 484$, Note sans date sur l'établissement des actes de notoriété pour les Marocains.

${ }^{72}$ Avis du Conseil d'État du 9 mai 1922, reproduit dans la documentation de la Résidence de Rabat, CADN, $1 / \mathrm{MA} / 200 / 484$.

${ }^{73}$ CADN, 1/MA/200/484, Avis reproduit du Conseil d'État, séance du 9 mai 1922.
} 
le taux de la pension doit-il être augmenté à proportion du nombre d'ascendants, ou celui-ci doit-il être compris dans un montant fixe ? Observant la législation tunisienne et en particulier le décret beylical du 18 août 1919, la section estime que la fixité et l'invariabilité du taux de pension « quelle que soit la composition de la famille indigène » est un principe intangible. On retrouve ici un motif colonial attaché à toutes les politiques publiques touchant à la famille musulmane : celle-ci semble apparaître comme une hydre au nombre de têtes indéterminé et sensément démesuré par rapport à une famille française (standard à partir duquel le législateur métropolitain légifère). Il convient alors à tout prix, pour des raisons utilitaristes d'économie, de ne jamais proportionner le montant d'une indemnité ou d'une pension au nombre des membres d'une famille, que le législateur craint toujours, sans information bien précise, pléthorique.

Cette situation aboutit, de l'aveu même des autorités coloniales, à des distorsions frappantes entre le droit métropolitain et le droit marocain, passé brutalement au tamis de sa prétendue coutume. En réalité, celle-ci est résumée au droit coranique de l'héritage en partant du principe erroné d'une application de plano des textes juridiques dans la réalité des sociétés musulmanes ${ }^{74}$. Ainsi de la veuve remariée dont le statut n'est pas défini en tant que tel dans l'école malikite et qui perd ainsi, dans le système des pensions militaires français, tout droit à pension d'un de ses fils morts ${ }^{75}$. D'après une lecture rapide des sources du droit, la mère est liée à ses enfants par rapport de tutelle affectueuse, dite hadana. Mais en cas de mort du mari, et de remariage de la mère, la veuve remariée perd tout droit sur ses enfants $m a h d \bar{u} n^{76}$. Elle ne pourra le recouvrer qu'en cas de nouveau veuvage. Si l'on suit le savoir orientaliste produit sur la famille musulmane, la veuve remariée perd donc tout intérêt à une pension, puisqu'elle n'a plus la charge des enfants. Dans le fond, en passant les « usages et coutumes » au filtre de ce savoir rigoureusement juridique, il faut bien constater avec la direction de l'intendance du Protectorat que les veuves militaires indigènes «ont été nettement défavorisées par rapport aux veuves des militaires français ${ }^{77}$.

Mais c'est à ce moment qu'il faut s'arrêter : faute d'études amples sur l'histoire sociale de la famille au Maroc, il est absolument impossible d'opposer une «vérité » à la «distorsion»

\footnotetext{
${ }^{74}$ Sur la valeur de cet écart, voir Mohammed Hocine Benkheira, Aver Giladi, Catherine Mayeur-Jaouen, Jacqueline Sublet, La famille en islam d'après les sources arabes, Paris, Les Indes Savantes, 2013.

${ }^{75} \mathrm{CADN}, 1 / \mathrm{MA} / 200 / 484$, Le Directeur de l'Intendance du Maroc au Directeur du Cabinet militaire et au Directeur des Affaires indigènes, 4 février 1930.

${ }^{76}$ Ernest Zeys, Traité élémentaire de droit musulman algérien (école malékite), Alger, Adolphe Jourdan, 1885 t. 1, p. 26.

77 CADN 1/MA/200/484, Le Directeur de l'Intendance du Maroc au Directeur du Cabinet militaire et au Directeur des Affaires indigènes, 4 février 1930.
} 
produite par la lecture coloniale. L'historien n'a pas le pouvoir de présenter une vérité positiviste contre des catégories administratives qui sont, comme dans toute politique publique, une reconstruction du réel. Il n'est pas possible, faute de données robustes, de mesurer le taux de pratique de la polygamie au Maroc. Au simple titre de suggestion, un document postérieur du Secrétariat général compte dans un service 191 agents mariés, dont 15 seulement seraient polygames ${ }^{\mathbf{7 8}}$. L'essentiel reste de souligner l'éminence de la «coutume» comme catégorie administrative dans le protectorat lyautéen sans chercher à la réduire en pièces à tout prix. Cette coutume est figée dans la pierre et a quelque chose de monolithique : faire des 'udūl des officiers d'état civil, cela serait heurter la coutume des Marocains, en conséquence il ne faut rien faire. Faire ainsi parler les Marocains à leur place en supposant leur indignation est un argument commode mais sans fondement particulier, tant la réforme de la justice religieuse fait l'objet de revendications des fonctionnaires marocains et du mouvement national. En cela, la «coutume musulmane» est une invention française, qui fabrique pour les Marocains leurs propres traditions ${ }^{79}$. Cette usurpation, cette façon d'occuper les cœurs des Marocains comme aurait dit Abdellah Hammoudi ${ }^{80}$, n'est pas qu'une opération de l'esprit mais contient en elle-même de lourdes conséquences politiques.

\section{Conclusion : le besoin inconcevable de la monarchie marocaine}

La chorégraphie lyautéenne est une séquence de communication politique qui, célébrant une union des cœurs autour de la guerre, ne fait que préparer un divorce qui dissout très vite ce bref mariage. Rétrospectivement, certains acteurs du mouvement national voient clair dans le jeu résidentiel et jugent sévèrement Moulay Youssef. En « envoyant des milliers de travailleurs et de paysans grossir les rangs de l'armée française (tadakhkhul fì șufüf al-junūd al-faransiyya) pour remporter la victoire pour le compte des Français (li-ḩisāb alfaransiyyinn) » le Sultan a incontestablement participé à ce que le nationaliste Muhammad alMakkī al-Nāṣirī qualifie d' «abolition du trône » (ilg $\bar{g} \bar{a}$ ' al- 'arsh $)^{81}$. La Grande Guerre, en prétendant animer la collaboration avec les élites musulmanes, renforce encore l'autoritarisme

78 CADN, 2/MA/1/49, Note au sujet du remboursement des Frais de voyage de congé en Algérie et en Tunisie, ler mars 1938.

79 Eric Hobsbawm et Terence Ranger, L'invention de la tradition, trad. par Christine Vivier, Paris, Éditions Amsterdam, 2006.

${ }^{80}$ Abdellah Hammoudi, op.cit., p. 151 et suiv.

${ }^{81}$ Muḥammad al-Makkī al-Nāșirī, Mawqif al-umma al-maǵribiyya min al-h̆imāya al-faransiyya [opinion de la nation marocaine sur le protectorat français], harakat al-waḥda al-maǵribiyya, 1946, p. 161. 
résidentiel au niveau des institutions politiques.

Au regard de l'application de cette politique publique, le Protectorat est apparu en prise avec son mauvais génie. Sollicitées par la métropole, par l'armée et par les anciens combattants, les autorités coloniales se heurtent à un obstacle qu'elles ont contribué à forger en refusant la simple idée qu'un ' $a d l$ puisse être un notaire et que le témoignage soit une preuve juridique. La « coutume » justifie, aux yeux des autorités coloniales, un gouvernement divisé entre État colonial et Makhzen. Mais la politique des pensions révèle le besoin du Makhzen pour gouverner le Maroc, besoin dont le Protectorat ne peut se défaire jusqu'à la Seconde Guerre mondiale, dont les pensions présentent les mêmes problèmes que dans les années 1920 et 1930 .

En cela, contrairement au récit incontournable du Protectorat déclinant dans l'administration directe après 1924, schéma qu'on trouve aussi bien chez Charles-André Julien que dans la prose hagiographique de Lyautey ${ }^{82}$, celui-ci avait posé les fondements du blocage futur de la situation coloniale. En bon scénographe, il est le principal auteur de cette distinction irréductible entre la «modernité » coloniale et la « coutume musulmane », il a forgé une logique de séparation entre ces deux sphères, qui sont aussi le double-visage de l'État, qui demeure jusqu'en 1956. À la fameuse circulaire du coup de barre, une circulaire symétrique se présente : celle du 11 juin 1922 sur l'enseignement musulman. Le maréchal y confie ses doutes sur la question des débouchés des Marocains dans la fonction publique (celle des cadres nouveaux et «modernes »), en la jugeant prématurée. Il estime que ceux-ci doivent bien, pour le moment, rester dans le sillon de leur tradition sans réclamer des diplômes français. Il juge l'exemple du fils du Pacha Bargache, gouverneur de Rabat, excellent : il a « développé chez nous sa culture, a appris notre langue et est rentré chez son père dans la norme de leur vie habituelle, et cela est très bien. Il n'a pas de baccalauréat ni d'équivalence et n'en a pas besoin $»^{83}$. Ici, le choix du Makhzen est ostensible, tout comme les résumés essentialistes de Lyautey. Mais ce que montre, en guise d'hypothèse, la politique des pensions c'est l'impossibilité radicale de séparer, comme le fait le Résident général, la modernité de la tradition, dans la mesure où sans cesse l'État colonial doit, au-delà des rubans décoratifs, recourir au Makhzen pour gouverner. À prendre Lyautey à la lettre, on finirait par croire, comme le Bloch de Proust, que la « vérité politique habite toujours, indiscutable et

\footnotetext{
82 Et qui consiste à présenter Lyautey comme l'auteur d'un chef d'œuvre dévoyé ensuite par ses prédécesseurs, en passant de l'admirable « contrôle » à l'administration directe.

${ }^{83}$ CADN,1/MA/200/388, examen de conscience du maréchal Lyautey, 11 juin 1922.
} 
matérielle, le dossier secret du président de la République et du Président du Conseil » alors que ces documents n'en offrent qu'un « cliché radioscopique ».

\section{Fonds d'archives}

Centre des archives diplomatiques de Nantes, France (CADN)

Archives du Ministère des Affaires étrangères, La Courneuve, France (AMAE)

Khizāna șbīhịyya, Bibliothèque Sbihi, Salé, Maroc

Archives du Maroc, Rabat, Maroc (AM) 